\title{
AN ADAPTIVE ANTENNA EMPLOYING GENETIC ALGORITHM
}

\author{
C. Laohapensaeng ${ }^{1}$, C. Free ${ }^{1}$ and M.Krairiksh ${ }^{2}$ \\ ${ }^{\mathrm{l}}$ MSRG Research Group \\ University of Surrey, Guildford GU2 7XH,UK \\ Email: c_laohapensaeng@hotmail.com \\ Tel. +4401483686108 , Fax. +4401483689404 \\ ${ }^{2}$ Faculty of Engineering and Research Center for \\ Communications and Information Technology \\ King Mongkut's hstitute of Technology Ladkrabang \\ Bangkok 10520, Thailand \\ Email: kkmonai@kmitl.ac.th
}

\begin{abstract}
This article simplifies the complexity of an adaptive antenna by employing the genetic algorithm. The system requires only the error signal and the phase-shifters in the discrete value to form an antenna pattern. An adaptive antenna with two elements and sixteen discrete steps of phase shifter is used. The instantaneous sample of an error signal is used as the cost function, which is minimized by using the genetic algorithm. Numerical results show that this antenna can improve signal to noise ratio in excess of $15 \mathrm{~dB}$.
\end{abstract}

\section{Introduction}

Nowadays, adaptive antenna plays a key role in improving the signal quality in the wireless communication by pointing main bearn of the antenna pattern toward the desired signal and placing nulls in the interference source directions. The famous articles that explain about an application of the adaptive antenna to the wireless communication have been pubiished by many authors such as Godara[1-2], and basic theoretical investigation can be found in $[3,4]$. Although the adaptive antenna can provide excellent performance, however it is very expensive and complicate in hardware design. Recently, R.L.Haupt [5] proposed an adaptive antenna employing the genetic algorithm. It has a unique feature that requires only the discrete phase-shifters to serve as the weights to form an antenna pattern. Thus, it leads us to the simple and low cost system. Nevertheless, this system has several limitations on some applications in which the direction of desired signal must be known. Therefore, it is not suitable for some applications, which are based on the low cost design and blind in direction of arrival of signal such as TV reception, smart cars, mobile handset and etc. In order to support these applications, this article extends the study on an adaptive antenna employing the genetic algorithm in blind direction environment.

\section{Adaptive antenna}

The diagram of an adaptive antenna using genetic algorithm (AAGA) and the coordinate system are shown in Fig. 1. It is formed by $N$ antennas along the $z$ axis which are used to feed the phase-shifters. Each of these phase-shifters has the value in discrete form. The microprocessor is used to calculate the certain discrete value of phase-shifters in order to form the antenna patterns. The cost function, which can be defined by several terms, must be used as the input data to the microprocessor for calculating the value of phase-shifters. In this work, the instantaneous square of the error signal $\left(\varepsilon^{2}(k)\right)$ is considered as the cost function and minimized by using the genetic algorithm [6].The advantage of AAGA over the typical adaptive antenna can be described as follow.

Typical algorithms for calculation of the value of weights are based on the gradient method, such as Least Mean Square (LMS). This algorithm requires the error signal as the cost function, which is obtained from the square of the difference between the reference signal and the array output signal in addition to the covariance matrix. It is formed from the 
signal received at each antenna element. Thus, it is very expensive due to the requirement the several high-speed analog to digital converters. In addition it requires the sophisticated calibration for the precision value of the complex weights. However, for the case of AAGA only the cost function (error signal) is required for calculating the value of weights and the precision of the value of the weight is not seriously restricted. Thus the sophisticated calibration is not required and results in the simple and low cost system design.

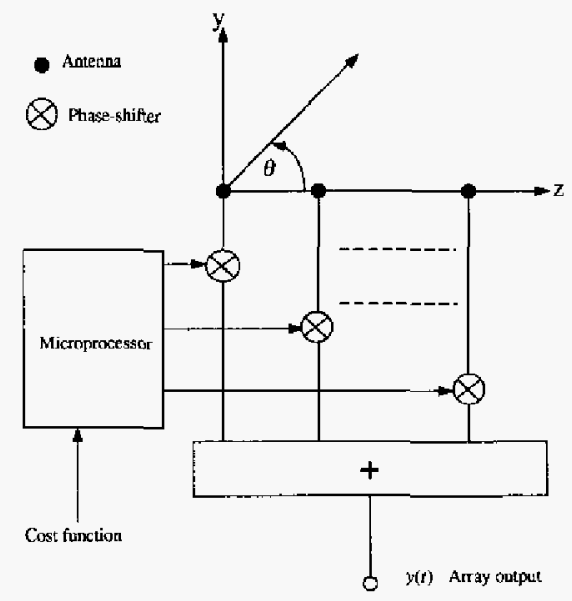

Fig.1 Diagram of an adaptive antenna

\section{Results}

To demonstrate the operation of the AAGA in the blind direction environment, two signals $S_{1}$ and $S_{2}$ are assumed to arrive the antenna in the same plane in the direction of 50 degree and 100 degree, respectively. In this assumption $S_{1}$ is the desired signal while $S_{2}$ is the interference signal. The power signal of $S_{1}$ is $3 \mathrm{~dB}$ higher than $S_{2}$. The number of array elements is two. The spacing between the elements is $0.5 \lambda$. The value of each discrete phaseshifters are divided into 16 steps. In the genetic algorithm, we begin with 8 chromosomes. In each chromosome there are 8 random bits. Only the four best chromosomes are kept for mating in the genetic algorithm process.

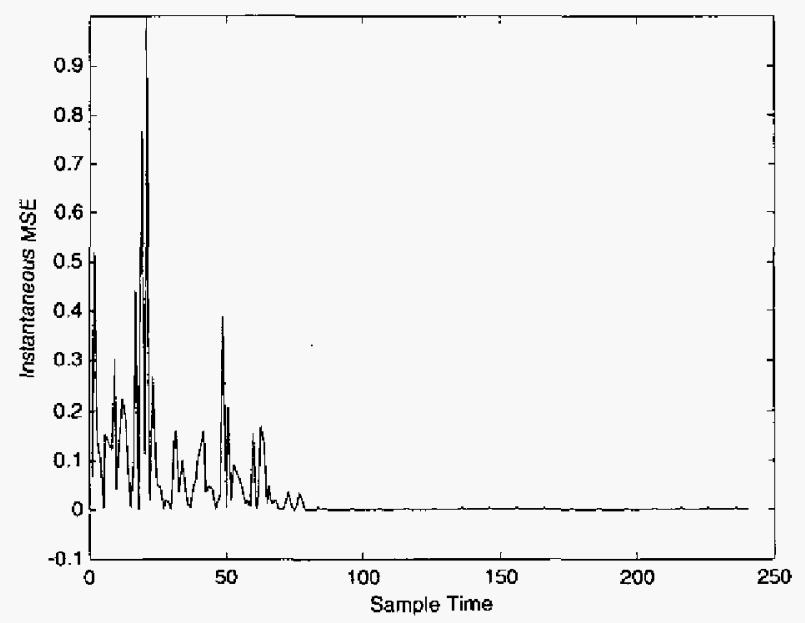

Fig.2 Error signal versus sample time. 
Figure 2 shows the $\varepsilon^{2}(k)$ (Instantaneous MSE) versus the sample time. It is obvious that after 80 samples or around $10 \mathrm{GA}$ generations of the genetic algorithm process, the optimum antenna pattern can be obtained. The value of $\varepsilon^{2}(k)$ is lower than $10 \%$. Nevertheless, it does not equal zero since the antenna has the limited value of the phase-shifter. Thus, it cannot completely form an antenna pattern such that placing the null in the direction of interference signal. Figure 3 shows the polar diagram of the antenna pattern when the sample time is 240 or $30 \mathrm{GA}$ generation. From the figure, the antenna can point the main beam toward the desired direction completely and place null slightly shift from the direction of interference signal due to the limited value of the phase-shifter as mentioned above. However, it indicates that the adaptive antenna employing genetic algorithm can improve the signal quality by trying to form an antenna pattern that reduces the interference signal.

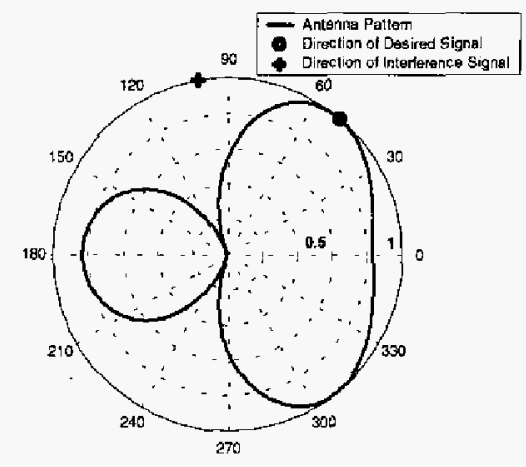

Fig.3 Anterna pattern of the $30^{\text {th }} \mathrm{GA}$ generation

To understand the characteristic of the adaptation of antenna pattern of the AAGA, Figure 4 shows the optimum antenna pattern of each GA generation versus the GA generation. It shows that an adaptive antenna begins with pointing main beam toward the undesired direction (100 degree) while placing null in the direction of desired source (50 degree). When the GA generation is around $10 \mathrm{GA}$ generation or 80 samples time the adaptive antenna can adapt the main beam toward the desired source.

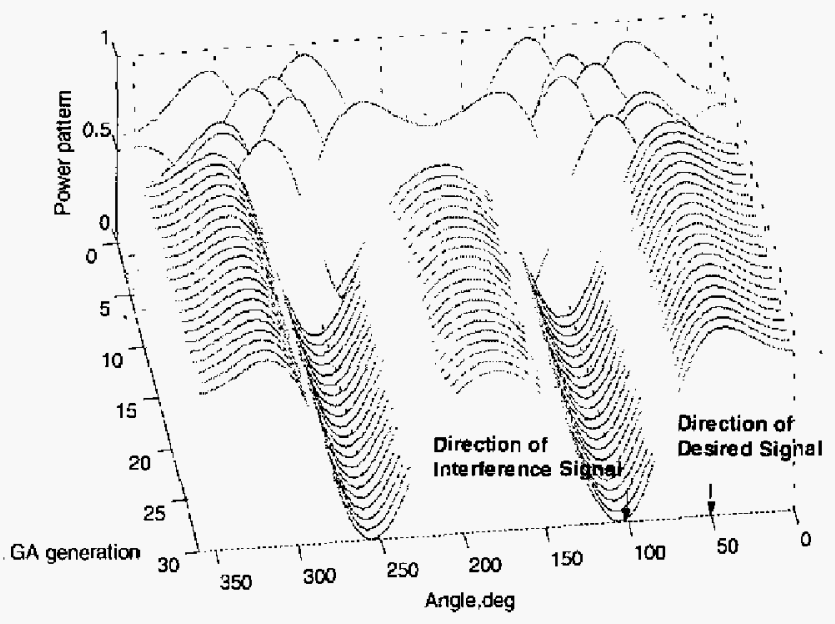

Fig.4 Antenna pattern versus the generation

One of the parameters which can explain the ability in receiving signal in any direction of the antenna is the antenna directivity [7]. Thus, in order to investigate the ability receiving signal 


\section{IEEE International Workshop on Antenna Technology}

of the adaptive antenna employing genetic algorithm. The term directivity ratio which is the ratio of directivity in the desired direction to the directivity in the interference direction is calculated. The graph of directivity ratio versus sample time is shown in Fig.5. We can see that the ability to receive. signal of the adaptive antenna in the desired direction is around $18 \mathrm{~dB}$ higher than interference direction after the adaptation of the antenna pattern is accomplished.

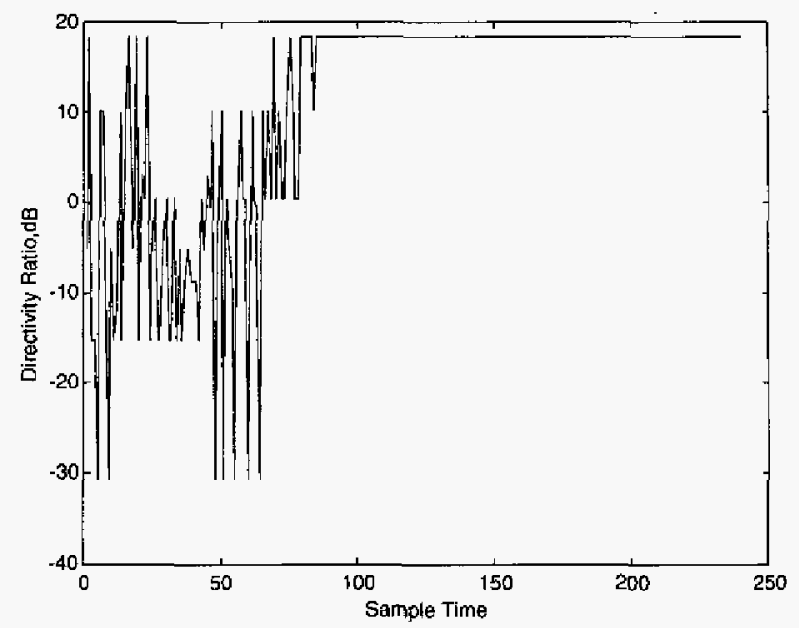

Fig.5 Directivity ratio versus the sample time

The improvement of the signal quality with the adaptive antenna can be expressed by the comparison between the signal to interference ratio (SIR) of the array output and the array input. The signal to interference ratio at the array output can be determined by multiplying the directivity ratio by the SIR of the array input (here is $3 \mathrm{~dB}$ ). Thus, the SIR of the array output is $21 \mathrm{~dB}$ and the improvement of the signal quality is $18 \mathrm{~dB}$, respectively.

\section{Conclusion}

This article presents the preliminary studies on an adaptive antenna employing genetic algorithm. This antenna system requires only array output to construct the cost function. In addition, phase-shifters in discrete value form are used. To investigate the operation of this antenna, an adaptive antenna with two elements and sixteen discrete steps of phase-shifters is considered. Two signals consisting of the desired signal and the interference signal are assumed to arrive the antenna in the same plane. Numerical results show that this antenna system provides a satisfactory performance in forming antenna pattern. After the antenna pattern forming is accomplished, the SIR is excess of $15 \mathrm{~dB}$ can be improved.

\section{References}

[1]L.C. Godara, "Appaication of Antenna Array to Mobile Communications, Part I: Performance Improvement, Feasibility, and system considerations," Proc. IEEE, vol.85, No. 4, pp. 1029-1060, July 1997.

[2] L.C. Godara, "Appdication of Antenna Array to Mobile Communications, Part II: BeamForming and Direction of Arrival Consideration," Proc. IEEE, vol.85, No. 4, pp. $1195-$ 1245, August 1997.

[3] J.E.Hudson, Adaptive Principle. Peter Peregrinus Ltd., 1991.

[4] R.T. Compton, Jr, Adaptive Antenna Concepts and Performance. Prentice Hall, 1988.

[5]R.L. Haupt, "Phase-Only Adaptive Nulling with a Genetic algorithm," IEEE Trans. Antennas Propagat., vol. 45, No. 6, pp. 1009-1015, June 1997.

[6]R.L. Haupt, "An irtroduction to genetic algorithms for electromagnetics," IEEE Antenna and propagation Magazine," wol. 37, No. 2, pp. 7-15, April 1995.

[7]C.A. Balanis, Antenna Theory Analysis and Design. John Wiley \& Sons, 1997. 